\title{
JÉRÉMIE FOA
}

\section{Banalité du corps \\ La prison, l'exil et la marche comme communauté d'expériences huguenotes au temps des guerres de Religion}

L'hypothèse que cette réflexion entend ici défendre peut se résumer en quelques mots: l'identité huguenote s'est avant tout constituée et consolidée par le biais des expériences corporelles auxquelles les huguenots ont été quotidiennement soumis au temps des guerres de Religion. En d'autres termes, je tenterai d'étudier un processus de socialisation corporelle, c'est-à-dire de mettre en lumière les mécanismes essentiels d'engendrement de l'habitus huguenot. Il s'agit de montrer que le média par excellence par lequel se sont intériorisées les identités confessionnelles, en l'occurrence l'identité huguenote, est moins le livre, la Bible, le pamphlet que le corps. Celui-ci sera étudié ici dans ses expériences quotidiennes. Je laisserai donc de côté la question des massacres et des violences (qui s'adressent pourtant bien aux corps) ${ }^{1}$ pour m'intéresser aux routines, aux expériences répétées que subissent les corps, au moyen d'une Alltagsgeschichte du temps des troubles ${ }^{2}$. À rebours de la philosophie politique qui fait de la guerre civile le »mal extrême«, on privilégiera ici la »banalité du mal«३3.

Ballotés par les vents de l'histoire, les corps huguenots sont sans cesse enfermés, affamés, arrêtés, déplacés, expulsés, exilés; quotidiennement, fouillés, suspectés, dépouillés mais aussi identifiés, reconnus, sentis et vus. Les corps sont de la sorte quotidiennement soumis à des »épreuves de vérité« dont la multiplication conduit à la fixation et à la reproduction des identités confessionnelles ${ }^{4}$ : comment traverser sans encombre un quartier catholique lorsqu'on est un piéton protestant? Où se rendre pour faire baptiser ses enfants ou inhumer ses proches? Qu'est-ce que passer des semaines voire des mois en prison siginifie-t-il pour »le faict de sa conscience«? Il y a là, dans la

1 Denis CRouZet, Les guerriers de Dieu. La violence au temps des guerres de Religion, vol. II, Seyssel 1991.

2 Carola LiPP, Histoire sociale et »Alltagsgeschichte«, dans: Actes de la recherche en sciences sociales 106-107 (1995), p. 53-66.

3 Nicolas Dubos, Le mal extrême. La guerre civile vue par les philosophes des origines à nos jours, Paris 2010. Je reprends ici, en le détournant, le titre d'une célèbre étude d'Hannah ARENDT, Eichmann à Jérusalem. Rapport sur la banalité du mal, Paris, ${ }^{2} 1991$.

4 Voir les réflexions sur une guerre civile contemporaine menées par Natalia SUAREZ BonILLA, La compétence du savoir-(sur)vivre. Épreuves d'identité dans la guerre civile colombienne, thèse de l'EHESS (2010) sous la direction de Luc Boltanski. Voir aussi les critiques adressées par Joan StAVO-DEBAUGE à Luc Boltanski quant à sa lecture de la guerre civile, dans: De la critique, une critique. Sur le geste »radical« de Luc Boltanski, EspacesTemps.net (http://www. espacestemps.net/en/articles/de-la-critique-une-critique-sur-le-geste-radical-de-luc-boltanski-2-en/ [consulté le 02/01/2014]). 
lignée de propositions avancées par Maurice Merleau-Ponty, une perspective de microhistoire sensorielle des guerres de Religion, une optique volontairement antiintellectualiste, qui postule que les cachots, les exils mais aussi les marches répétées pour se rendre au temple dans les faubourgs ont autant fait pour la construction de l'identité huguenote que les textes de Calvin. Il s'agit donc d'inverser la perspective culturaliste. Non pas partir d'une identité toute faite qui aurait jeté ces hommes dans des expériences similaires, mais au contraire partir des épreuves vécues pour entrevoir la construction de l'identité. À défaut de connaître une expérience de la communauté, qui leur était de fait interdite, les protestants français ont d'abord vécu une communauté d'expériences dont le corps (privé de liberté ou soumis à d'interminables marches) a été le média privilégié.

\title{
PRISES DE CORPS: LA BANALITÉ DES FERS
}

\author{
Voilà comment de nous la verité bannie, \\ Meurtrie et deschirée, est aux prisons, aux fers, \\ Ou esgare ses pas parmi les lieux deserts 5 .
}

L'emprisonnement est sans doute l'expérience la mieux partagée par les protestants français. Bien que l'incarcération ne soit en théorie pas une peine mais le temps d'une attente de jugement ${ }^{6}$, elle est, dans les faits, très souvent utilisée comme une mesure tout à la fois punitive et préventive à l'encontre des huguenots. Quelques exemples permettront d'illustrer ce propos. La détention des protestants en temps de guerre est, dans les villes catholiques, une mesure courante et fréquemment employée pour se prémunir contre d'éventuels »ennemis de l'intérieur«. Les listes de »suspects« que confectionnent patiemment les gouverneurs servent, une fois l'urgence venue, à se saisir au plus vite des réformés sur le pas de leur porte. En juillet 1570, au cours de la troisième guerre civile, François Porchier, avocat réformé au bailliage de Mâcon, est jeté en prison »où il auroit demeuré avec les aultres de la religion sept sepmainnes entieres, sans pouvoir estre eslargys, encores qu'ils n'ussent en rien forfaict et delinqué et qu'ils heussent presenté plusieurs requestes pour l'elargissement de leurs personnes « ${ }^{7}$. Si les villes ayant massacré leurs concitoyens protestants lors de la SaintBarthélemy sont relativement rares (à l'échelle du royaume), très nombreuses sont en revanche celles qui, à cette occasion, ont mis les huguenots sous les verrous, tout à la fois pour s'en préserver et pour les protéger. À Lisieux, le gouverneur Carrouges ordonne dès le 28 août 1572 l'emprisonnement de tous les réformés ${ }^{8}$. Le comte de Charny enjoint au maire de Dijon »de se saisir des personnes de ceulx de ladicte religion et

5 Agrippa D’AubignÉ, Les Tragiques, s.1. 1616: Princes, vers 162-164, p. 52.

6 Camille DÉGEZ, Un univers carcéral $\left(\mathrm{XVI}^{\mathrm{e}}-\mathrm{XVII}{ }^{\mathrm{e}}\right.$ siècle) la prison de la Conciergerie et sa société, thèse de l'École des chartes (2005) sous la direction de Denis Crouzet.

7 Archives municipales (AM) Mâcon, GG 123, pièce 13.

8 AM Lisieux, BB 7, fol. 342. 
habitans de la ville, mesmes des plus apparentz, qui ont faict faction et exercice d'icelle religion, donné aide, conseil faveur, presté argent pour leur faction « ${ }^{9}$. Même protocole à Issoire, Montferrand ou Clermont en Auvergne. Dans ces dernières villes, comme dans de très nombreuses autres, l'emprisonnement sauve la vie des huguenots en les défendant contre la furie des massacreurs. À Chalon-sur-Saône, les habitants catholiques vont jusqu'à assurer »que ceux de la nouvelle religion se sont vouluntairement renduz prisonniers sans aucune emotyon ny difficulté, tellement que quelques ung ont estés receus à tenir prison ès maisons d'aucungs catholicques, gens de bien, qui en respondront au peril de leurs vyes $^{10}$. Pourtant, dans d'autres villes, l'emprisonnement préventif des huguenots fonctionne comme une souricière pour ces derniers et les »massacres aux prisons«, comme l'a montré Denis Crouzet, constituent bien souvent la »troisième phase « des tueries ${ }^{11}$. À Meaux, Rouen, Lyon ou Gaillac, c'est aux prisons que se commettent les violences. Privés de liberté, de cachettes et d'armes, les corps huguenots sont des proies d'autant plus vulnérables.

Caractéristique de la »petite guerre«, la pratique de l'emprisonnement des ennemis est loin de cesser avec la paix. En janvier 1571, six mois après l'édit de Saint-Germain, Henri de Navarre doit encore écrire au gouverneur de Guyenne pour lui demander la libération d'Hélye Granger, prisonnier »pour le fait de la religion «12. Le cas du parlement de Paris, au cœur de mes recherches récentes, le montre bien. Il y a bien là une expérience durable des huguenots sur le temps long des dernières décennies du $\mathrm{XVI}^{\mathrm{e}}$ siècle. À l'heure de la paix, nombre de réformés sont toujours derrière les fers. À défaut de faire exécuter les réformés, comme il le faisait quelques années auparavant, faute de pouvoir les jeter en prison, le parlement de Paris les y maintient bien après la conclusion des édits de pacification. Autrement dit, mauvaise volonté et lenteur administrative se conjuguent pour retenir des centaines de huguenots derrière les barreaux plusieurs mois après la paix. Ainsi, nonobstant l'édit d'Amboise (enregistré par la cour parisienne le 27 mars 1563), on trouve encore de nombreux protestants engeôlés. Quelques exemples suffiront à le montrer. Ce n'est que le 8 mai 1563 que le premier prisonnier huguenot inculpé pour »fait de religion« est libéré. Encore doit-il subir une véritable humiliation, contraire au texte de l'édit de paix: Nicolas Letellier, orfèvre de Porcy »prisonnier ès prisons de la conciergerie du Palais à Paris pour le fait de la nouvelle secte et opinion [...] auroit declairé qu'il vouloit aller à la messe, vivre et se gouverner selon les institutions de l'église catholique et romayne«. Il est élargi à condition de »faire profession de sa foy catholique «13. Retenu plus longtemps que la loi ne le demandait, le corps du protestant est ici assujetti, par une institution royale, à l'effectuation d'un rituel d'abjuration contraire à la loi royale. Letellier n'est en rien

9 AM Dijon, B 208, fol. 15v.

10 AM Chalon-sur-Saône, EE 1 (6 septembre 1572).

11 Crouzet, Les guerriers de Dieu (voir n. 1), p. 110.

12 Recueil de lettres missives d'Henri IV, éd. par M. Berger DE Xivrey, Paris 1843, vol. I, p. 14

13 Archives nationales (AN), X2a 31, fol. 21; 8 mai 1563 (Parlement criminel, registre d'arrêts transcrits). 
une exception. Tous les huguenots emprisonnés pour »fait de conscience« - certains ne seront libérés qu'en 1564 (!) - doivent se soumettre à la même palinodie de papier.

Le vocabulaire utilisé par la cour pour dire son opposition au protestantisme est tout aussi révélateur: en cette même date du 8 mai 1563, le parlement libère Jehan Millet, porcher natif de Tours, qu'il dit être touché de la »nouvelle secte et hérésie«. Lui aussi n'est élargi qu'à condition de se faire absoudre des »excommuniemens qu'il a encourus $^{14}$. En ces heures où la défense de l'honneur conduit souvent à la rixe mortelle ${ }^{15}$, le choix des mots par le parlement n'est pas anodin - il s'agit bien de blesser par l'usage des mots ${ }^{16}$. Ce vocabulaire injurieux, contraire à toute la nomenclature officielle que la cour est pourtant censée distribuer (religion prétendue réformée) n'est abandonné par le parlement qu'au mois de juillet 1563 .

Quelques exemples ne prétendant nullement à l'exhaustivité permettent de montrer que ces cas ne sont en rien isolés mais se répètent, rendant l'expérience de l'emprisonnement (légal comme illégal) commune aux huguenots du royaume. Le 8 mai 1563, quelques heures après les faits cités précédemment, six huguenots sont de nouveau libérés, avec les mêmes exigences de se »conserver« en la religion catholique, apostolique et romaine. Le 12 mai, la cour élargit Pierre Dathie, prisonnier en la conciergerie »à la charge de se faire absouldre des censures par luy encourues [...] et de faire profession de sa foy par devant l'evesque de Senlis [...] et de vivre catholiquement «17. Tout au long de l'année 1563, c'est ainsi une petite centaine de prisonniers huguenots qui est relâchée au compte-goutte, toujours à condition de faire profession de foi catholique. À Bordeaux, des réformés emprisonnés pour fait de religion présentent encore requête aux commissaires de l'édit pour obtenir leur libération le 6 septembre $1563^{18}$ soit six mois après la conclusion de la paix d'Amboise. L'emprisonnement est ce faisant une expérience corporelle, fréquente pour les huguenots du temps des troubles.

Puisqu'il est difficile de les incarcérer pour des faits de conscience, en théorie protégés par les édits, la violation de l'édit est un prétexte aisé pour les expédier en prison. En mai 1572, plusieurs dizaines de réformés de Troyes sont emprisonnés »pour avoir esté au presche audict Ysle«, c'est-à-dire hors du lieu de culte qu'il leur a été officiellement octroyé19. La prison, on le sait, est un univers difficile pour ceux qui y séjournent. Les plus riches peuvent aisément acheter les éléments d'un confort spartiate auprès de leurs geôliers pour qui la prison est un métier, ou mieux, une »affaire« comme une autre. Mais pour les plus humbles, la faim et le froid harcèlent sans répit les corps. À Chalon-sur-Saône, les habitants catholiques se demandent que faire de

14 Ibid., fol. 21.

15 Michel Nassiet (éd.), Les lettres de pardon du voyage de Charles IX (1565-1566), Paris 2010.

16 AN, X2a 131, fol. 21.

17 AN, X2a 31, fol. 33 (12 mai 1563).

18 Bibliothèque nationale de France (BNF), Manuscrits français (Ms. fr.) 15878, fol. 130.

19 AM Tours, BB 14, pièce 43; 29 mai 1572 (Lettre du duc de Guise à la municipalité de Tours). Sur la question des prêches, Jérémie FOA, An Unequal Apportionment. The Conflict over Space Between Protestants and Catholics at the Beginning of the Wars of Religion, dans: French History 20 (2006), p. 369-386. 
"plusieurs de ladicte nouvelle religion qu'ilz sont si pauvres et necessiteux qu'ilz meurent de faim en prison, n'ayant de quoy vivre, sinon au jour la journée «20. L'incarcération participe ce faisant à la construction d'un stock de souffrances partagées; épreuve initiatique, blessure de guerre ou marque des braves, elle contribue à forger un ensemble d'expériences communes que ni des conditions sociales hétéroclites, ni une origine géographique, ni même des croyances religieuses encore flottantes (en 1560) ne fournissaient aux huguenots du royaume. Le "protestant français«, s'il fallait l'essentialiser, est un ancien détenu ou un futur captif. »La communauté d'expérience se substitue à l'expérience de la communauté 21 $^{1}$.

Non moins fréquent, l'exil est la deuxième »prise« sur le corps que cet article entend analyser, en ceci qu'il est un vecteur décisif d'intériorisation d'une identité dominée.

\section{EXILS}

Pendant les guerres, parfois longtemps après la conclusion de la paix, les huguenots sont nombreux à éprouver en leur corps l'expérience de l'exil. La grande variété des mots témoigne de la fréquence de l'épreuve du déracinement. Le vocabulaire pour désigner les exilés dépend pour l'essentiel du point de vue adopté et de la façon dont

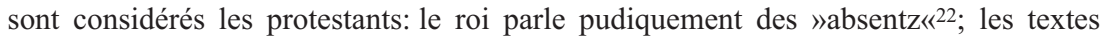
mentionnent joliment les »forissites«. Fourquevaux dit de Vieilleville qu'il a remis $»$ les fuitifz de Provence en leurs maisons $23^{23}$; l'intransigeante mairie de Dijon parle quant à elle des »expulsez« ${ }^{24}$. À l'inverse, les protestants de Carcassonne se présentent comme »bannis et chassez $^{25}$. Les commissaires évitent presque toujours de parler de »fuitifs" et préfèrent aussi parler d'expulsés. C'est le cas d'Anthoine Fumée et de Jacques Viart, qui écrivent au roi avoir réintégré dans la ville de Moissac »ceux de la religion qu'on dict reformée, qui en avoyent esté expulsez«²6.

Partout, les conséquences spatiales des guerres de Religion se lisent dans l'homogénéisation confessionnelle et la fermeture des villes qu'elle entraîne: presque partout, les minorités religieuses sont exclues et entament la ronde des exils, l'appropriation des uns passant par l'expropriation des autres. En juillet 1562, les échevins de Cler-

20 AM Chalon-sur-Saône, EE 1, pièce non numérotée (septembre 1572). Sur ce point, Jérémie FOA, »Bien unis et paisibles«? Une »non Saint-Barthélemy« à Chalon-sur-Saône (septembre 1572), dans: Véronique CASTAGNET, Olivier CHRISTIN, Naïma GHERMANi (dir.), Les affrontements religieux en Europe, du début du XVI ${ }^{\mathrm{e}}$ au milieu du XVII ${ }^{\mathrm{e}}$ siècle, Lille 2008, p. 217-229.

21 Pierre BourdieU, Abdelmalek SAYAD, Le déracinement. La crise de l'agriculture traditionnelle en Algérie, Paris ${ }^{2} 2002$, p. 136.

22 AM Aix, BB 59, fol. 48 (1563).

23 Edmond CABIÉ (éd.), Guerres de Religion dans le Sud-Ouest de la France et principalement dans le Quercy, d'après les papiers des seigneurs de Saint-Sulpice de 1561 à 1590, Albi, Paris 1908 , p. 29.

24 AM Dijon, D 63, pièce non paginée.

25 Archives départementales (AD) Gard, C 925, pièce 1 (automne 1563).

26 BNF, ms. fr. 15879, fol. 57 (21 février 1564). 
mont ordonnent »à tous seditieux et toutes personnes qui sont de la nouvelle secte et religion « de »vuider« la ville dans les trois jours ${ }^{27}$. Ailleurs, dans un chassé-croisé de la proscription, les catholiques chassés de la ville de Blaye occupent à Bordeaux les maisons des protestants expulsés ${ }^{28}$. Par la suite, le retour huguenot n'a rien d'un long fleuve tranquille et les »absents «, comme on les appelle pudiquement, doivent attendre des mois l'arrivée des commissaires du roi pour pouvoir regagner leurs foyers. À Moissac, ce n'est qu'en février 1564, onze mois après la conclusion de la paix d'Amboise, que les commissaires de l'édit réintègrent »ceux de la religion qu'on dict reformée, qui en avoyent esté expulsez «29. Les protestants de Mâcon, expulsés en février 1562, ne se présentent aux portes de leur ville que le 8 juin 1563, plusieurs mois après la paix. Encore ne sont-ils pas reçus immédiatement, la municipalité catholique faisant les plus grandes difficultés à les laisser entrer: les gardes verrouillent les portes et veillent au loin sur la troupe des exilés, qui se résout à camper pour lors dans la »prayerie« 30 . À Langres, les catholiques épaulés par le chapitre déploient une inentamable énergie à entraver le retour des huguenots dans la ville entre 1562 et $1566^{31}$.

Loin du confort de la ville, de la routine du foyer et de la chaleur des familles, les huguenots bannis, et parmi eux les plus notables, font ainsi en leur corps l'expérience de la dramatique réversibilité de l'ordre social ${ }^{32}$. Le retour d'exil ne signe en rien l'effacement du traumatisme. De fait, »on « ne rentre jamais et c'est toujours un autre qui rentre, changé, bouleversé33. S'il rentre, car certains ne reviennent jamais et s'installent tout bonnement ailleurs, quand ils le peuvent, espérant trouver là des cieux plus accueillants. Anthoine Gairard, »maistre cousturyer de la ville de Cordes en Albigeoys«, s'installe à Albi avec »sa femme et enfens«. Mais ailleurs, on n'est rarement bien reçu. Les consuls d'Albi tentent de l'expulser, avec d'autant moins de scrupules que l'»estrangier « qu'il est se double d'un huguenot ${ }^{34}$. En outre, le »lieu« qu'on retrouve n'est demeuré intact que dans les souvenirs de l'exilé. Pendant le temps de l'absence, la ville ou le village ont changé, certains ont prospéré, d'autres ont fui ou sont morts. Il est fréquent, de retour d'exil, de trouver ses proches déguerpis ou, plus souvent encore, sa maison occupée, son office usurpé. Les huguenots de Toulouse poursuivent pendant des années la récupération de leurs maisons, vendues à l'occasion de leur exil au temps des premiers troubles ${ }^{35}$. Les huguenots Jehan Le Normant et

27 AM Clermont(-Ferrand), BB 33; 8 juillet 1562.

28 Archives historiques du département de la Gironde, XII, 1870, p. 62.

29 BNF, ms. fr. 15879, fol. 57 (21 février 1564).

30 BNF, ms. fr. 4048, fol. 147 (8 juin 1563). Sur Mâcon, pendant les guerres de Religion, Ami Bost, Histoire de l'Église protestante de Mâcon, Mâcon 1977.

31 AD Haute-Marne, 2 G 136, quatre pièces (1562-1566).

32 Sur la question de la »réversibilité de l'ordre social«, Cyril LemIEuX, De la théorie de l'habitus à la sociologie des épreuves. Relire »L'expérience concentrationnaire«, dans: Liora ISRAËL, Danièle Voldman (dir.), Michael Pollak. De l'identité blessée à une sociologie des possibles, Paris 2008, p. 179-205.

33 Günther ANDERS, Journaux de l'exil et du retour, Paris 2012.

34 AM Albi, GG 79, pièce non paginée.

35 AM Toulouse, AA 15, fol. 139 (février 1571). 
Jehan Chartier, au lendemain de l'édit d'Amboise »seroient retournez en leurs maisons pour faire l'exercice de leurs estats et vivre en pais«. Mais de retour chez eux, ils trouvent leurs biens dispersés, leurs maisons gardées par des hommes d'armes et leurs »estats« usurpés ${ }^{36}$.

$\mathrm{Au}$ travers de cette expérience d'exil, le corps quitte les repères familiers et les certitudes rassurantes qui l'accompagnent. Les huguenots de Mâcon se décrivent comme ayant erré des mois parmi des »terres étrangères«. Sur qui compter quand amis et familles sont restés au pays? Comment manger loin de son métier? Le corps acquiert alors d'autres compétences, celles qui permettent de survivre en milieu inconnu, il change ses habitudes, modifie ses projets. La guerre civile engendre un savoir(sur)vivre. Certains réformés expulsés de Mâcon sont devenus brigands, tant la rupture avec »l'allant de soi«, avec les normes sociales hier approuvées, a été radicale. Il s'ensuit une modification durable des manières d'être et des sensibilités. C'est souvent l'occasion d'une prise de conscience des protestants comme collectif. Dans plusieurs villes, c'est le cas à Mâcon, le retour en groupe des huguenots permet la maturation d'une conscience collective: en rentrant ensemble, on fait masse, on se compte, on se voit, on parle et l'on prie ensemble. Des corps rentrant d'exil forment à cette occasion un corps. Pourtant, dans de nombreux cas, cette communauté d'expériences ne forge pas une expérience de la communauté. Très souvent en effet, le »départ« et le »retour« relèvent de décisions individuelles. Les mieux informés, les plus aisés aussi n'attendent pas l'arrêté d'expulsion collectif pour fuir et comprennent d'eux-mêmes qu'il est temps de partir: à Lyon, la protestante Isabel Moreaux a tôt l'intuition qu'avec le déclenchement de la troisième guerre civile, les équilibres ont basculé à Lyon et qu'il faut s'exiler ${ }^{37}$. Jehan Chastel »belotier et passementier « de Toulouse ${ }^{38}$, s'est »retiré« à Paris lors des derniers troubles "parce que les habitans de Thoulouse le menassoient de le massacrer et tuer parce qu'il est de la religion nouvelle $\ll^{39}$. Bien que vécues par plusieurs, ces épreuves sont avant tout celles d'un individu ou d'une famille.

Surtout, dans de très nombreux cas, les villes catholiques n'acceptent le retour des huguenots qu'à condition que celui-ci se fasse au goutte à goutte et non massivement. À Aix-en-Provence, le commissaire royal décide que les réformés »seront conduictz en leursdictes maisons par petites troupes, en nombre de vingt, trente, quarente ou autre plus petit nombre par gens notables qui seront par [lui] pour ce commis et deputez « ${ }^{40}$.

36 AN, X2a 131, fol. 29 (11 mai 1563).

37 AD Rhône, BP 318, Audiences de la sénéchaussée (décembre 1568-octobre 1569), non folioté.

38 Joan DAVIES, Persecution and Protestantism: Toulouse 1562-1575, dans: Historical Journal 22 (1979), p. 121-134.

39 AM Toulouse, BB 113 (police des capitouls), non folioté, à la date (23 janvier 1571).

40 AD Bouches-du-Rhône (Annexe d'Aix), B 3648, fol. 1169, »faict au conseil tenu à Aix le huictiesme aoust MDLXIII [1563]«. Une chronique contemporaine rapporte ainsi l'ordre de Vieilleville »que les réformés qui avoient quitté leurs maysons pour cause de religion, seroient restablis et réintégrés dans leurs biens, offices et estats; qu'ils seroient pour cela reconduits par petites troupes chez eux, pour y demourer sous la saulvegarde de l'auctorité publique, qui leur garantiroit, avec la sûreté personnelle, la liberté de conscience«, dans Gustave LAMBERT, Histoire des guerres de Religion en Provence, vol. I, Nyons 1972, p. 203. 
Non seulement les protestants ne peuvent, comme le font les ecclésiastiques qui reviennent dans les villes protestantes, occuper symboliquement lors de leur rentrée l'espace dont on les avait chassés mais encore sont-ils reconduits gardés et flanqués par quelque notable local. S'agit-il de les contrôler ou de les protéger? De s'assurer qu'ils ne sortiront pas du rang ou qu'on ne leur fera aucun mal? De la sorte, on interdit précisément ce qu'on avait permis aux ecclésiastiques: faire de leur marche de rapatriement une manifestation de triomphe, c'est-à-dire transformer en prise de possession urbaine la fin d'une expulsion humiliante. Si l'on considère les regroupements (ou leur absence) comme des projections dans l'espace de réalités sociales, l'incapacité huguenote à faire groupe vient signifier leur incapacité politique à faire corps, c'est-à-dire à être représentés au niveau des instances urbaines - leur émiettement spatial dit alors leur dispersion, leur seule existence individuelle, et partant leur inexistence corporative et politique.

De fait, ces expulsions répétées impliquent une surveillance particulière des huguenots, des dispositifs moins policiers qu'administratifs qui enserrent les huguenots dans des réseaux de papiers, autant de »fils invisibles« (Karl Marx) qui se transforment en chaînes véritables une fois la guerre revenue. Ils relient entre eux et malgré eux des huguenots qui souvent ne se connaissent pas mais sont unis en une communauté de destins. Ces fils invisibles apparaissent à partir de 1562 et se généralisent en 1568: il s'agit des listes de »suspects de la nouvelle religion« qui sont établies dans de très nombreux villes et villages du royaume. Elles permettent en temps voulu de savoir qui est huguenot et de le mettre rapidement hors d'état de nuire ${ }^{41}$. Elles serviront du reste lors des massacres de la Saint-Barthélemy. Ainsi, à Clermont en Auvergne, dès 1567, une première liste de protestants, pointant les hommes qui avaient pris les armes contre le roi, comportait vingt-trois noms ${ }^{42}$. Elle est réactualisée en 157443 . On trouve des listes similaires à Riom, mais aussi à Toulouse en $1568^{44}$ ou à Dijon à la même date ${ }^{45}$. Elles facilitent l'expulsion, le massacre (on marque d'une croix les maisons des hommes à liquider), mais aussi l'enfermement à domicile. Elles précipitent une prise de conscience, matérialisent en la couchant sur le papier l'existence d'un groupe insé-

${ }^{41}$ Le sujet des listes sous l'Ancien Régime est une thématique encore trop méconnue, pour laquelle je renvoie aux résultats à venir du programme collaboratif »Pour une histoire des listes« dirigé par Grégoire Salinero et Christine Lebeau (EA 127-CRHM).

42 AM Clermont, BB 40, non folioté (30 janvier 1574). Cette liste, reprise en 1574, avait été dressée sur ordre du gouverneur, Saint-Hérem, en décembre 1567 (AM Clermont, BB 36, non folioté, 18 décembre 1567 »a esté exposé avoir reçeu commandement de Monseigneur de Sainct Heran [lieutenant général d'Auvergne] de fere rolle de tous les suspectz de la religion nouvelle de la present ville; affin que lesdits suspectz ne soyent employés par cy après à la garde des portes $\ll$ ).

43 Georges Rouchon, Listes des protestants de Clermont (Auvergne) 1567-1574, dans: Revue d'Auvergne XXXVIII (1921), p. 145-158.

44 Paul Romane-Musculus, Les protestants de Toulouse en 1568, dans: Bulletin de la Société de l'histoire du protestantisme français 107 (1961), p. 77.

45 Edmond BELLE, Études sur la Réforme et les guerres de Religion en Bourgogne. La Réforme à Dijon des origines à la fin de la lieutenance genérale de Gaspard de Saulx-Tavannes (15301570), Paris 1911. 
parable du danger qu'il charrie. Elles relient entre elles, par leur matérialité même, papier ou parchemin, des individus qui n'étaient pas forcément rattachés. En même temps, l'objectivation des noms permet l'accumulation du savoir sur les suspects hors des corps de leurs voisins; loin d'être exclusivement manipulables par les autochtones, ces listes peuvent ce faisant être mobilisées par des hommes extérieurs au village. Elles signalent donc quelque chose qui est en train d'échapper à la communauté dans la mesure où elles trahissent l'impossibilité de connaître par cœeur (ou par corps) tous les hérétiques du quartier: à quoi bon sinon coucher sur le papier la litanie des noms de suspects s'il est si facile de s'en souvenir? La liste signale donc ce point de bascule où tout le monde ne connaît plus tout le monde, c'est-à-dire le passage de la communauté à la sociétét6. Elle précipite une autonomisation du savoir sur les suspects que n'accompagne pas encore, au temps des guerres de Religion, un processus d'autonomisation des institutions en charge de la répression. Le moment venu, il revient encore aux voisins, aux divers capitaines, penons, quarteniers et dizainiers de se saisir de leurs prochains protestants ${ }^{47}$.

En juin 1568, les échevins de Clermont menacent de »harquebouzer« tous les protestants qui »seroient trouvés hors de leur domicile«. Autant d'obstacles à la liberté de circulation des corps huguenots qui freinent la constitution des collectifs protestants, d'autant plus qu'il leur est interdit de s'assembler. Dans le même sens, il est relativement rare de rencontrer des quartiers proprement huguenots dans la France des guerres de Religion, alors qu'il y a des quartiers ouvertement catholiques. Pour l'essentiel, l'expérience de la communauté et la prise de conscience de l'existence d'un corps huguenot vont se faire à travers les déplacements répétés auxquels les corps huguenots sont soumis pour se rendre sur leurs lieux de culte. Il y a là sans doute l'expérience fondamentale du corps protestant comme média d'intériorisation d'une identité dominée.

\section{LA MARCHE AU TEMPLE}

Les édits de pacification (notamment celui d'Amboise en mars 1563 et celui de SaintGermain en août 1570) sont en effet loin de régler l'ensemble des questions posées par la coexistence confessionnelle. Si la loi précise que les huguenots doivent être mis en possession des faubourgs d'une ville par bailliage pour y faire leur culte, elle ne précise pas laquelle: s'agira-t-il de la ville principale? Au lendemain des conflits, en 1563 et 1570, après consultation, et sans doute négociation, est établie une première liste de villes aux faubourgs desquels le culte huguenot est autorisé. Dans ces listes, la ville

46 Les développements qui suivent s'inspirent de Robert DESCIMON, Qui étaient les Seize? Étude sociale de 225 cadres de la Ligue radicale parisienne (1585-1594), Paris 1983 (Mémoires publiés par la Fédération des sociétés historiques et archéologiques de Paris et de l'Île-de-France, 34), p. 264.

47 Jack Goody, La logique de l'écriture. Aux origines des sociétés humaines, Paris 1986 p. 172. 
principale du bailliage ou du gouvernement est rarement choisie. Il suffit pour s'en convaincre d'évoquer les villes prévues en Normandie: Pont-Audemer, Caudebec, Vire, Conches, Gisors, Carentan, Alençon. En 1564, les huguenots du bailliage de Senlis, doivent se rendre aux faubourgs de la ville d'Allonne (à $58 \mathrm{~km}$ de distance) pour faire leur culte public ${ }^{48}$. Il y a bien là une expérience corporelle, pédestre, décisive pour les protestants français. Des raisons stratégiques et militaires sont certes à l'œuvre mais les raisons symboliques paraissent plus fortes encore. En parcourant de manière quotidienne ou hebdomadaire la distance physique qui les sépare de leur prêche, les corps huguenots parcourent la distance sociale et religieuse qui les sépare de l'ordre légitime. L'incorporation par les huguenots de leur exclusion s'accomplit ainsi pas à pas, au ras du sol, la distance spatiale traduisant directement une distance sociale et religieuse ${ }^{49}$. Le corps en est le média privilégié.

Au demeurant, rien n'étant moins fixe que l'attribution de ces lieux, leur localisation est soumise à de fréquentes pressions, à de multiples suppliques et pétitions, qui occasionnent de nombreux »changements d'adresse« et témoignent de l'inégale capacité des deux confessions à mobiliser des appuis en leur faveur. Si le conseil royal tranche en dernier ressort, de nombreuses instances - gouverneurs, nobles, commissaires royaux, municipalité, clergé - peuvent être mobilisées pour faire pencher la balance au profit de l'un ou de l'autre groupe, c'est-à-dire éloigner ou rapprocher le prêche. Mais ici encore, protestants et catholiques sont inégalement armés, en particulier à cause de l'intercession presque systématique des gouverneurs, très hostiles aux protestants dans leur majorité. En Champagne, alors que le roi avait proposé la ville de Chaumont en Bassigny pour y faire le culte, le gouverneur, un Guise, choisit une localité »à l'extrémité du gouvernement dedans les Ardennes à quatorze grandes lieuës de la ville de Chaumont $\ll^{50}$. En revanche, à Montdidier, où les protestants ont un puissant protecteur en la personne de Louis de Condé, gouverneur de Picardie, qui par mariage possède de nombreuses terres dans les environs, ils obtiennent le culte aux faubourgs ${ }^{51}$.

Aussi l'importance des réseaux conditionne-t-elle pour beaucoup l'attribution des lieux de culte huguenots. Par cette voie, les catholiques s'assurent la désignation des villes aux faubourgs desquelles le culte réformé est autorisé. À l'inverse, les protestants se plaignent de n'avoir été »receus à en nommer aucune «52. En outre, la nécessaire maîtrise des outils juridiques et l'indispensable dotation en moyens financiers redoublent la dépossession des huguenots. À la différence des catholiques en effet, et

48 Pasteur BonET-MAURY, Les origines de la Réforme à Beauvais, dans: Bulletin de la Société de $1^{\prime}$ histoire du protestantisme français 23 (1874): $\mathrm{n}^{\circ} 1$, p. 73-88; $\mathrm{n}^{\circ} 2$, p. 124-137, 217-232, ici p. 134.

49 Pierre Bourdieu, Effets de lieu, dans: ID. (dir.), La misère du monde, Paris, 1993, p. 249-262, ici. p. 260.

50 Lettre de Monseigneur le Prince de Condé à la Roine mere du Roy, dans: Denis-François SECousse (éd.), Mémoires de Condé. Servant d'éclaircissement et de preuves à l'Histoire de M. de Thou, contenant ce qui s'est passé de plus mémorable en Europe [...], 6 vol., Londres 1743-1745, vol. IV, p. 275.

51 Victor DE BEAUVILlÉ, Histoire de la ville de Montdidier, vol. II, Paris ${ }^{2} 1875$, p. 200.

52 Lettre de Monseigneur le Prince de Condé (voir n. 50), p. 275. 
particulièrement là où ils sont minoritaires, ils ne peuvent mobiliser les ressources juridiques et financières que procure par exemple la mainmise sur un échevinage, le contrôle d'un présidial ou d'un parlement. C'est manifeste à Dijon où le parlement délègue en la personne de Bégat un de ses plus brillants représentants pour s'opposer à l'édit d'Amboise ${ }^{53}$. C'est évident à Tours où les échevins paient des avocats, envoient des délégués en cour ou ailleurs pour refuser la création d'un prêche dans les faubourgs de leur ville. En 1564, ils paient par exemple douze écus à Gilles du Vierger, avocat, pour avoir tenté d'»empescher l'establissement des presches que ceulx de la nouvelle religion demandoient en l'un des forsbours de cestedite ville, [et avoir] faict et redigé par escript plusieurs plaidoiez remonstrances et memoires « ${ }^{54}$. En 1570, ils délèguent deux hommes à La Rochelle auprès du maréchal de Cossé, en charge de l'application de l'édit, »pour assister à l'establissement qui se debvoit faire pour ledict seigneur du presche general de ceux de la nouvelle religion du gouvernement de Touraine et pour luy nommer lyeu propre et plus élongné de la ville de Tours que fayre se pouvoir « 55 .

Ainsi, la capacité d'un groupe à obtenir un lieu de culte proche (c'est-à-dire à maîtriser la localisation de l'espace qui est attribué à son corps) tout autant que son pouvoir de tenir à distance les personnes indésirables et les constructions qui les accompagnent (temples, cimetières), dépendent fortement de la dotation de ce groupe en capital, social, symbolique mais aussi financier. Autrement dit, la distribution inégalitaire des lieux de culte trahit l'inégale dotation en capital des différents groupes religieux et peut être lue comme la traduction spatiale de cette inégalité. Là où les protestants sont nombreux, sont riches, ont des appuis ou tiennent un consulat, ils peuvent rapprocher leur lieu de culte. À l'inverse, là où ils sont faiblement dotés, leur prêche est éloigné et implanté dans des espaces stigmatisés où se concentrent les propriétés négatives. À Troyes par exemple, les huguenots doivent se contenter du lieu de Séant en Othe ${ }^{56}$, »meschante petite ville« selon Pithou. Les huguenots s'assemblent dans une grange à Gaillac, un grenier à Angers, à l'hôpital des pestiférés à Carcassonne ${ }^{57}$. À Lyon, les protestants qualifient le bourg de Quincieu qui leur a été attribué de »vieille motte de terre « ${ }^{58}$. Des espaces sans identité accueillent une identité sans espace. Ce faisant, les luttes pour la maîtrise de l'espace sont loin de n'être que des disputes insignifiantes ou des simples querelles de préséance. Elles engagent au contraire des questions vitales, à

53 Jean Agnault BÉGAT, Remonstrances au Roy des députez des trois Estats de son duché de Bourgoigne sur l'édict de la pacification, par où se monstre qu'en un royaume deux religions ne se peuvent soustenir, et les maulx qui ordinairement adviennent aux roys et provinces ou les hérétiques sont permis et tolérez, Anvers 1563.

54 AM Tours, CC 79, fol. 69.

55 AM Tours, CC 88, fol. 111 (27 septembre 1570).

56 Séant-en-Othe, aujourd'hui Bérulle, à $38 \mathrm{~km}$ à l'est de Troyes.

57 R. P. Bouges, Histoire ecclésiastique et civile de la ville et diocèse de Carcassonne. Avec les pièces justificatives et une notice ancienne et moderne de ce diocèse, Péronnas 1994 ( $1^{\text {re }}$ éd. 1741).

58 AM Lyon, GG 77, pièce non paginée. 
proprement parler, puisqu'il y va à la fois de la sauvegarde terrestre et de la survie dans l'au-delà mais aussi de la reproduction sociale du groupe.

\section{CONCLUSION}

Loin d'affadir les identités religieuses, la cohabitation de deux confessions distinctes dans l'espace tend à exacerber les signes extérieurs de distinction entre protestants et catholiques $^{59}$. Les corps sont le théâtre de ces stratégies d'affrontement: mise en prison, exil et marches façonnent les consciences en sculptant les corps. La coexistence confessionnelle, c'est-à-dire la cohabitation quotidienne, suscite une comparaison et une compétition permanente dont les clercs sont loin d'être les seuls acteurs et la théologie loin d'être le seul champ. Le corps est en effet le moyen par excellence des stratégies de distinction. Dans son usage de l'espace urbain, le corps ennemi est soumis à des épreuves de vérité à travers lesquelles il est sans cesse assigné à sa confession: ouvrira-t-il sa boutique les jours fériés? Sortira-t-il de la ville le dimanche pour aller au prêche? Tapissera-t-il sa façade? L'intériorisation d'une identité confessionnelle passe donc par son extériorisation, à travers les multiples épreuves spatiales auxquelles les esprits mais surtout les corps (qu'on pense aux déplacements mais aussi à l'injonction de chanter à voix basse ou à celle de manger de la viande à Carême en secret) sont soumis quotidiennement et qui, répétées indéfiniment, finissent par devenir une habitude voire une identité. Cet usage des marqueurs spatiaux et corporels dans un but distinctif ou apologétique montre qu'on ne saurait oublier l'espace comme instrument essentiel du processus d'intériorisation et de solidification des identités religieuses. Autrement dit, le corps, comme sujet que l'on montre ou comme objet que l'on exhibe, constitue une dimension fondamentale du processus de construction confessionnelle.

\section{RÉSUMÉ}

L'hypothèse que cette réflexion entend défendre ici peut se résumer en quelques mots: l'identité huguenote s'est avant tout constituée et consolidée par le biais des expériences corporelles auxquelles les huguenots ont été quotidiennement soumis au temps des guerres de Religion. En d'autres termes, cet article étudie un processus de socialisation corporelle, c'est-à-dire qu'il met en lumière les mécanismes essentiels d'engendrement de l'habitus huguenot. Il s'agit de montrer que le média par lequel se sont intériorisées les identités confessionnelles, en l'occurrence l'identité huguenote, est moins le livre, la Bible ou le pamphlet que le corps. Celui-ci est étudié ici dans ses expériences quotidiennes. La question des massacres et des violences (qui s'adressent pourtant bien aux corps) est donc laissée de côté. Nous nous intéresserons aux routines, aux expériences répétées que subissent les corps, au moyen d'une Alltagsgeschichte du temps des troubles: exils, emprisonnements, éloignements, détours, marches au temple, cimetières déplacés,

59 Cf. les conclusions d'Étienne FranÇOIS, Protestants et catholiques en Allemagne. Identités et pluralisme, Augsbourg, 1648-1806, Paris 1993, p. 243. 
rues interdites, portes fermées, etc. À rebours de la philosophie politique qui fait de la guerre civile le »mal extrême«, nous privilégierons ici la »banalité du mal«.

\section{SUMMARY}

The hypothesis the present article intends to defend can be summed up as follows: Huguenot identity is generated and stabilised first and foremost by means of the physical experiences to which the Huguenots have been subjected on a daily basis in the times of the Wars of Religion. In other words, this article examines a process of physical socialization, which means bringing to light the main mechanisms by which Huguenot habitus is created. It seeks to show that the prime medium whereby confessional identities, in this case Huguenot identity, have been internalized is the body rather than the book, the bible, or the pamphlet. The body will be studied here in the ways it is experienced in daily life. The question of massacres and violence (though aimed at bodies) is thus left out in favour of routines, the repeated experiences to which bodies are subjected, by means of an »Alltagsgeschichte « (history of everyday life) of troubled times: exile, imprisonment, expulsion, detours, walks to the Temple, displaced cemeteries, prohibited roads, closed gates, etc. The term »banality of evil« is preferred here, inverting political philosophy which makes the civil war the »ultimate evil«. 